\title{
COMPARAÇÃO ENTRE FRAGMENTOS OBTIDOS COM AGULHAS DE CALIBRES 14 E 12 EM “CORE BIOPSY" ESTEREOTÁXICA DE LESÕES MAMÁRIAS IMPALPÁVEIS: DIFERENÇAS ENTRE O TAMANHO DOS FRAGMENTOS E FREQÜÊNCIA DOS TIPOS DE LESÕES DIAGNOSTICADAS*
}

\author{
Maria do Carmo Abreu-e-Lima1, Norma Maranhão², Virginia Almeida ${ }^{3}$, Cláudia Batista Melo ${ }^{4}$, \\ Ênio Araújo ${ }^{5}$, Manuela Abreu-e-Lima ${ }^{6}$, Adonis R.L. de Carvalho ${ }^{7}$
}

Resumo Com o objetivo de avaliar o desempenho das agulhas de calibres 14 e 12 na obtenção de "core biopsies" da mama sob controle estereotáxico, os autores compararam biópsias de lesões mamárias impalpáveis obtidas com agulhas desses calibres quanto ao tamanho dos fragmentos e freqüência dos tipos de lesões diagnosticadas. De um total de 1.009 biópsias, os autores analisaram 900 obtidas por agulhas 14 e 109 obtidas por agulhas 12, para análise da freqüência das lesões. Os tamanhos dos fragmentos foram analisados nos 700 primeiros casos para os espécimes obtidos por agulhas 14. A utilização de agulhas 12 resultou em maior tamanho dos fragmentos ( $p<0,001$ ), ausência de material insatisfatório, redução na proporção das lesões benignas não-neoplásicas $(p=0,03)$ e aumento na freqüência de carcinomas $(p<0,001)$, tanto in situ como invasivos. Tais achados podem refletir aumento da acurácia do diagnóstico, em virtude da maior quantidade de material e/ou melhor seleção radiológica das biópsias obtidas por agulhas 12, pela utilização coincidente da mamografia digital em campo limitado (estereotaxia digital).

Unitermos: "Core biopsy" estereotáxica. Câncer de mama. Agulha calibre 14. Agulha calibre 12.

\begin{abstract}
Comparison between the fragments obtained by stereotactic core biopsy of nonpalpable breast lesions using 14- and 12-gauge needles: differences on the length of the fragments obtained and on the frequency of the types of diagnosed lesions.

The purpose of this study was to compare the performance of 14- and 12-gauge needles for obtaining biopsy specimens under stereotactic guidance. The authors compared the biopsies of nonpalpable breast lesions using these different size needles, regarding the length of the fragments obtained and the types of diagnosed lesions. A total of 1,009 samples were obtained using 14-gauge (900 samples) and 12-gauge (109 samples) needles. The size of the samples was evaluated on the first 700 consecutive biopsies using 14-gauge needles. The use of 12-gauge needles consistently provided larger size specimens than 14-gauge needles $(p<0.001)$; a reduction in the prevalence of benign nonneoplastic lesions $(p=0.03)$ and a higher frequency of in situ and invasive carcinomas $(p<0.001)$ were also observed. These findings may suggest a higher diagnostic accuracy of biopsies performed with 12-gauge needles due to the larger samples obtained and/or due to a better radiological selection of those lesions, which were detected by digital mammography and biopsied under digital stereotactic guidance.
\end{abstract}

Key words: Stereotactic core needle biopsy. Breast cancer. 14-gauge needle. 12-gauge needle.

\footnotetext{
Trabalho realizado na Clínica Radiológica Lucilo Maranhão e no Laboratório de Patologia Adonis Carvalho, Recife, PE.

1. Professora Adjunta Doutora do Departamento de Patologia da Universidade Federal de Pernambuco (UFPE), Recife, PE.

2. Radiologista da Clínica Radiológica Lucilo Maranhão, Doutora pela Universidade Federal do Rio de Janeiro (UFRJ), Rio de Janeiro, RJ.

3. Patologista, Departamento de Patologia do Hospital de Câncer de Pernambuco.

4. Professora Assistente, Departamento de Patologia da UFPE.

5. Acadêmico de Medicina da UFPE.

6. Acadêmica de Medicina da Universidade de Pernambuco (UPE).

7. Professor Emérito, Departamento de Patologia da UFPE.

Endereço para correspondência: Profa. Dra. Maria do Carmo Abreu-e-Lima. Rua Pacífico dos Santos, 63, conj. 101. Recife, PE, 52011-030. E-mail: acarvalho@nlink.com.br

Aceito para publicação em 26/6/2001.
}

\section{INTRODUÇÃO}

A possibilidade de se estabelecer um diagnóstico anatomopatológico correto e conclusivo depende, primordialmente, da representatividade da amostra enviada para exame histopatológico. Em lesões mamárias impalpáveis essa representatividade foi viabilizada pelo desenvolvimento das técnicas de localização precisa, guiadas por métodos de imagem, ultra-sonografia e a estereotaxia, que asseguraram o diagnóstico mediante punção aspirativa por agulha fina (PAAF) ou de pequenos fragmentos ob- tidos por agulhas mais calibrosas ("core biopsy")(1). Todavia, a certeza do alvo atingido não é a única condição sine qua non para um diagnóstico preciso; para tanto, a amostra deve ser suficiente em tamanho e número de fragmentos. É sabido que a possibilidade de um diagnóstico conclusivo, subtipagem, gradação histológica e definição de invasão melhoram com o maior volume do tecido. Assim, são mais freqüentes casos de material considerado insuficiente para o diagnóstico nas preparações citológicas obtidas por PAAF, como também é menos precisa a definição de subtipagem 
histológica nesse tipo de espécime. Outra dificuldade inerente ao exame citológico consiste na distinção entre neoplasias in situ e invasiva.

Nath et al. demonstraram, em material obtido por meio de punção-biópsia diretamente a partir de peças cirúrgi$\operatorname{cas}^{(2)}$, melhor definição das lesões à medida que se aumenta o calibre das agulhas $(18,16$ e 14$)$.

A biópsia estereotáxica no diagnóstico das lesões subclínicas da mama foi desenvolvida visando, principalmente, evitar biópsia cirúrgica em pacientes com lesões benignas e, ao mesmo tempo, permitir um diagnóstico definitivo de câncer por meio de métodos não-cirúrgi$\cos ^{(3)}$. Na quase totalidade da literatura especializada os autores utilizam pistolas automáticas de longo alcance e agulhas calibre 14 para biópsia de fragmentos, com resultados satisfatórios.

\section{MATERIAL E MÉTODOS}

Este trabalho baseia-se no estudo de 1.009 biópsias percutâneas de fragmentos de lesões mamárias impalpáveis obtidas pelo método estereotáxico ${ }^{(4-10)}$ na Clínica Radiológica Lucilo Maranhão e examinadas no Laboratório de Patologia Adonis Carvalho, Recife, PE, durante o período de maio/1993 a outubro/2000.

A localização, inicialmente, foi feita por unidade estereotáxica (Stereotix 2) acoplada a um mamógrafo (Senographe DMR). Em 1999 foi introduzido o sistema digital direto em campo limitado da mama ("spot" digital), destinado à realização do método estereotáxico (G Stereotix Seno-Vision), que permite maior número de informações da imagem, pela possibilidade de ajustar brilho, contraste, utilização do "zoom" e inversão. O tempo de reconstrução da imagem é quase real (dez segundos) após a exposição dos raios X, proporcionando maior rapidez, menor tempo de exposição e, desse modo, a dose de radiação é reduzida para a paciente. Após a reconstrução, a imagem disponibilizada no monitor pode ser manipulada eletronicamente, mediante a utilização de vários recursos como inversão, "zoom" e lente eletrônica, para obter melhor qualidade na visibilização de detalhes, sem a necessidade de submeter a paciente a novas exposições.

Os fragmentos foram retirados com agulhas calibre 14 acopladas a pistola automática de longo alcance (Magnum 2,2 cm - Barp). A partir de janeiro/1998, iniciamos a utilização de agulhas calibre 12 para biópsia de fragmentos, em grande parte dos casos subsequientes (109 casos). Do número total de casos (1.009), estudamos, inicialmente, as 700 primeiras biópsias, todas obtidas com agulhas calibre 14 , correspondentes a 646 pacientes examinadas no período de maio/1993 a dezembro/1996. Excluímos, preliminarmente, um caso de mama masculina (ginecomastia) e 37 biópsias em que as amostras incluíram apenas pele e/ou tecido adiposo normal sem representação do corpus mammae, que consideramos inadequados para a finalidade desta pesquisa. Esses 37 casos foram utilizados como subgrupo para comparação. Das 662 biópsias restantes, utilizamos as 567 primeiras, obtidas até maio/ 1996, para avaliação da sensibilidade e especificidade do diagnóstico $(90,72 \%$ e $98,36 \%$, respectivamente, com teste de correlação de Spearman de 0,92$)^{(7)}$. O total geral (662 biópsias) obtido até dezembro/1996 foi utilizado para a verificação do tamanho dos fragmentos obtidos com agulhas calibre 14 . No material obtido por agulhas calibre 12 (109 biópsias) foram também analisados os tamanhos dos fragmentos. Na totalidade dos casos foi determinada a frequiência das lesões diagnosticadas pela "core biopsy" (1.009) e também, separadamente, para as "core" obtidas por agulhas calibres 14 (900 casos) e 12 (109 casos), respectivamente. Para o estudo comparativo, usamos o teste $t$ de Student e o teste do quiquadrado.

Na presença de microcalcificações os espécimes foram rotineiramente radiografados. Recentemente, temos adotado a pintura com nanquim para marcar os fragmentos que contêm as microcalcificações. Os fragmentos foram colocados sobre papel de filtro e processados como de rotina, embebidos em parafina e corados pela hematoxilina-eosina. Para a análise da frequiência das lesões encontradas, os casos foram separados em quatro cate- gorias: benignos não-neoplásicos, neoplasias benignas, lesões "borderline" e carcinomas. A categoria "benigno nãoneoplásico" enquadra grande variedade de lesões, inclusive as alterações funcionais benignas (displasia/doença fibrocística), processos inflamatórios e reacionais, linfonodos intramamários, etc.

Foram definidas como "borderline" as hiperplasias ductais atípicas e casos suspeitos de carcinoma mas sem elementos histológicos suficientes para o estabelecimento de diagnóstico conclusivo.

\section{RESULTADOS}

\section{Análise dos fragmentos}

Os fragmentos obtidos a "core biopsy" foram, na maioria, irregularmente alongados ou filiformes, de consistência elástica, cor branco-acinzentada, pardo-acastanhada ou diferentes mesclas dessas tonalidades. No exame macroscópico foi observado que alguns desses fragmentos filiformes eram maiores, de consistência mais firme e, em geral, não se fragmentavam no processamento histológico, mantendo seu formato e tamanho originais. Com muita frequiência estes correspondiam ao diagnóstico histológico de carcinoma ductal invasivo com acentuada fibrose (padrão cirroso). Na análise das 700 primeiras biópsias, todas obtidas por agulhas 14 , o material foi considerado insuficiente para o diagnóstico em 37 casos, correspondendo a $5,29 \%$ do total. Esses espécimes excluídos (37) resultaram em amostras com um a oito fragmentos, média de $3,89 \pm 1,91$. O comprimento do maior fragmento variou de 0,10 a $1,20 \mathrm{~cm}$, média de $0,42 \pm 0,21 \mathrm{~cm}$.

As "core biopsies" incluídas no presente estudo (662 casos) resultaram na obtenção de um a 14 fragmentos (média de $4,57 \pm 1,82$ ). O comprimento do fragmento maior variou de 0,22 a $3,00 \mathrm{~cm}$, com média de $0,75 \pm 0,47 \mathrm{~cm}$.

A Tabela 1 compara o comprimento do fragmento maior no material incluído no estudo e nos casos considerados insuficientes para o diagnóstico.

As diferenças entre esses dois grupos, quanto ao tamanho do maior fragmento, foram altamente significativas ( $p<0,001$ ). As 109 biópsias obtidas com agulhas ca- 
libre 12 constituíram entre um e 26 fragmentos, com média de 8,37 $\pm 4,05$. O comprimento do maior eixo do fragmento variou de 0,7 a $4,5 \mathrm{~cm}$, com média de 1,76 $\pm 0,67 \mathrm{~cm}$. A Tabela 2 compara esses fragmentos com aqueles obtidos por agulhas 14. As diferenças foram altamente significativas $(\mathrm{p}<0,001)$. Nesse material não ocorreram espécimes considerados insuficientes para o diagnóstico.

As Figuras 1 e 2 mostram a diferença, à microscopia, no tamanho das amostras obtidas por agulhas calibres 14 e 12 (mesmo aumento, isto é, $25 \times$ ).

\section{Análise da freqüiência das lesões encontradas}

A freqüência das lesões diagnosticadas por "core biopsy" pode ser vista no Gráfico 1, para a totalidade dos casos (1.009), englobando o período de maio/ 1993 a outubro/2000. Na casuística global (Gráfico 1), as lesões benignas corresponderam a 775 casos $(76,8 \%)$, predominando as lesões não-neoplásicas com 630 casos $(62,4 \%)$. A Tabela 3 compara o número e o porcentual das lesões nas amostras obtidas com agulhas calibres 14 (primeira coluna) e 12 (segunda coluna). Pode-se notar um predomínio das lesões benignas $(\mathrm{A}+\mathrm{B})$ e, dentre elas, as não-neoplásicas (A), tanto nos casos obtidos por agulhas 14 - com um total de 709 lesões benignas $(78,7 \%)$ e 583 nãoneoplásicas $(64,7 \%)-$, como naqueles obtidos por agulhas 12 - com um total de 66 lesões benignas $(60,5 \%)$ e 47 nãoneoplásicas $(43,1 \%)$.

Embora a diferença entre a proporção de lesões benignas $(\mathrm{A}+\mathrm{B})$ obtidas com agulhas $14(78,7 \%)$ e $12(60,5 \%)$ tenha sido de baixa significação $(\mathrm{p}=0,126)$, a diferença na proporção de benignos nãoneoplásicos (A) foi significante ( $\mathrm{p}=$ 0,03 ), de $64,7 \%$ e $43,1 \%$, respectivamente, para as agulhas 14 e 12 .

Os carcinomas corresponderam a 200 casos $(19,8 \%)$ da casuística total (Gráfico 1), com predomínio das formas invasivas, com 167 casos (16,5\%), sobre as in situ, com 33 casos $(3,2 \%)$. Nas amostras oriundas de agulhas 14, os carcinomas constituíram 159 casos $(17,6 \%)(\mathrm{D}+\mathrm{E})$, sendo $134(14,8 \%)$ invasivos e $25(2,7 \%)$ in situ (Tabela 3 ). Os espécimes obtidos

Tabela 1 Comparação entre o comprimento dos fragmentos maiores obtidos por agulhas 14 incluídos no estudo e os considerados insuficientes para o diagnóstico.

\begin{tabular}{|l|c|c|}
\hline \multicolumn{1}{|c|}{ Categoria } & Comprimento do fragmento maior & ${ }^{-} \mathrm{X} \pm$ desvio padrão \\
\hline Incluídos & 0,22 a $3,0 \mathrm{~cm}$ & $0,75 \pm 0,47 \mathrm{~cm}$ \\
Excluídos & 0,1 a $1,2 \mathrm{~cm}$ & $0,42 \pm 0,21 \mathrm{~cm}$ \\
\hline
\end{tabular}

$p<0,001$.

Tabela 2 Comparação entre o comprimento dos fragmentos maiores obtidos por agulhas $14 \mathrm{e}$ 12.

\begin{tabular}{|c|c|c|}
\hline Categoria & Comprimento do fragmento maior & $-{ } \pm$ desvio padrão \\
\hline 14 & 0,22 a $3,0 \mathrm{~cm}$ & $0,75 \pm 0,47 \mathrm{~cm}$ \\
12 & 0,7 a $4,5 \mathrm{~cm}$ & $1,76 \pm 0,67 \mathrm{~cm}$ \\
\hline
\end{tabular}

$p<0,001$.

Tabela 3 Comparação entre a freqüência das lesões identificadas nas amostras obtidas por agulhas 14 e 12 .

\begin{tabular}{|l|c|c|}
\hline \multirow{2}{*}{ Diagnóstico } & \multicolumn{2}{|c|}{ Calibres das agulhas } \\
\cline { 2 - 3 } & 14 & 12 \\
\hline A - Benignos não-neoplásicos & $583(64,7 \%)$ & $47(43,1 \%)$ \\
B - Benignos neoplásicos & $126(14 \%)$ & $19(17,4 \%)$ \\
(A+B) & $709(78,7 \%)$ & $66(60,5 \%)$ \\
C - "Borderline" & $32(3,5 \%)$ & $2(1,8 \%)$ \\
D - Carcinoma invasivo & $134(14,8 \%)$ & $33(30,2 \%)$ \\
E - Carcinoma in situ & $25(2,7 \%)$ & $8(7,3 \%)$ \\
(D + E) & $159(17,6 \%)$ & $41(37,6 \%)$ \\
\hline Totais & 900 & 109 \\
\hline
\end{tabular}

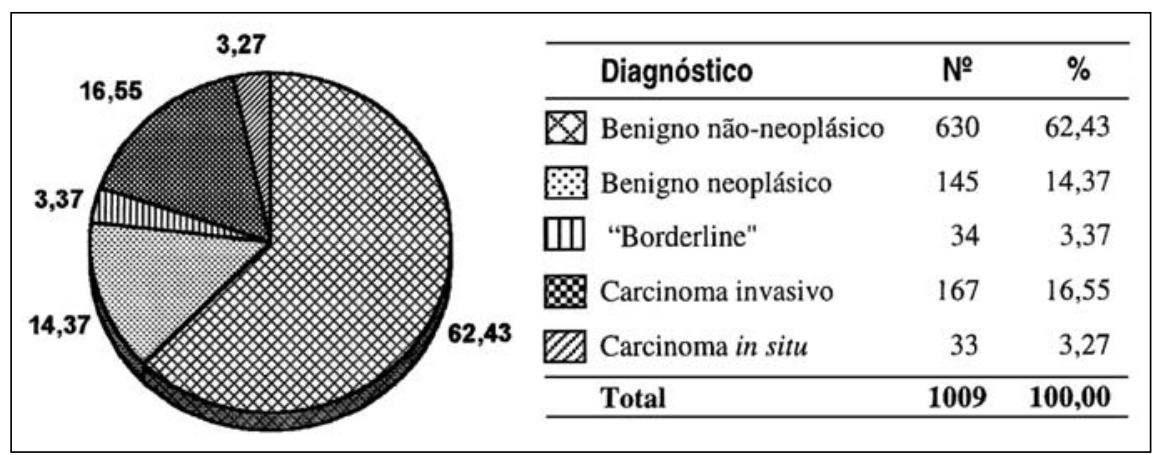

Gráfico 1. Freqüência dos tipos de lesões identificadas pela "core biopsy" em 1.009 casos (maio/ 1993 a outubro/2000).

com agulhas 12 resultaram em 41 casos $(37,6 \%)(\mathrm{D}+\mathrm{E})$ de carcinomas, sendo invasivos em 33 casos $(30,2 \%)$ e in situ em oito casos $(7,3 \%)$.

A comparação da freqüência de carcinomas no material obtido com agulhas $14(17,6 \%)$, com os mesmos dados para agulhas $12(37,6 \%)$, resulta em diferença altamente significante $(\mathrm{p}<0,001)$. Do mesmo modo, foi significante $(\mathrm{p}=0,03)$ a diferença na proporção de carcinomas in situ, quando se comparou sua freqüência nos espécimes obtidos por agulhas 14 , com 25 casos $(2,7 \%)$, e por agulhas 12, com oito casos (7,3\%); o mesmo ocorreu em relação à diferença na proporção dos carcinomas invasivos, com 134 $(14,8 \%)$ para os espécimes obtidos por agulhas 14 e $33(30,2 \%)$ para os obtidos por agulhas $12(\mathrm{p}=0,002)$. 


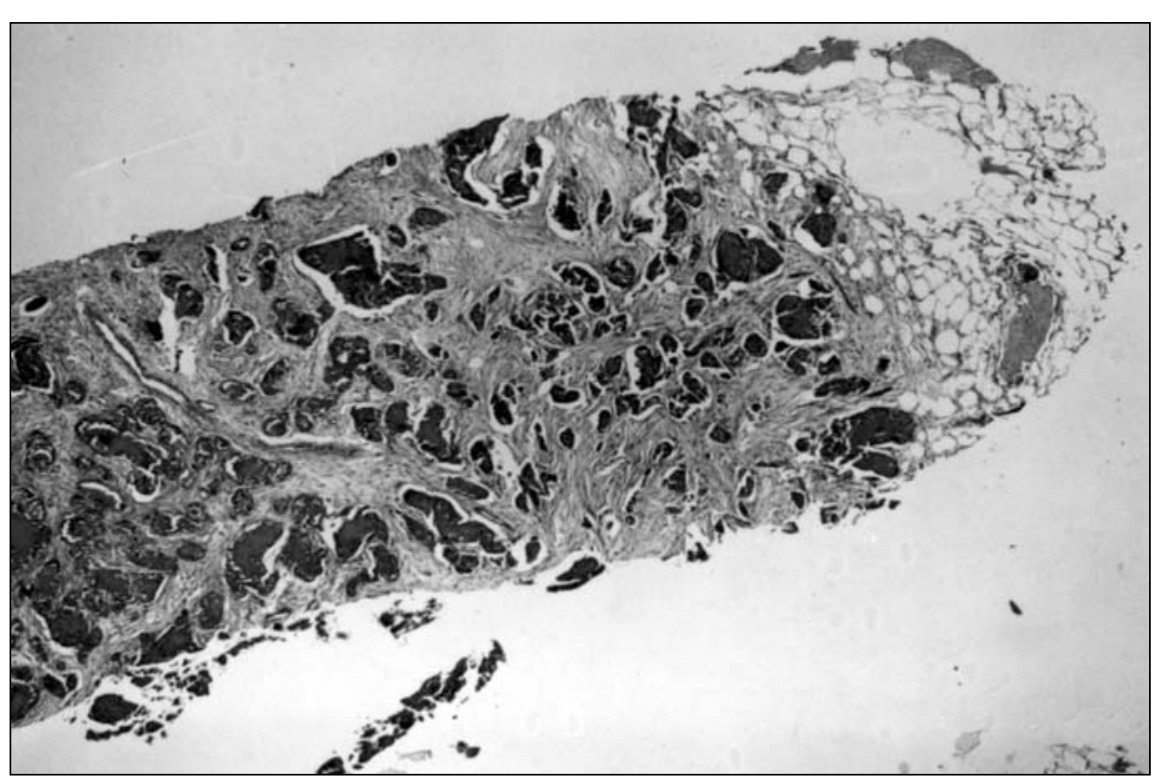

Figura 1. "Core biopsy", agulha 14. Carcinoma ductal invasivo. (Hematoxilina-eosina, original $40 \times$ ).

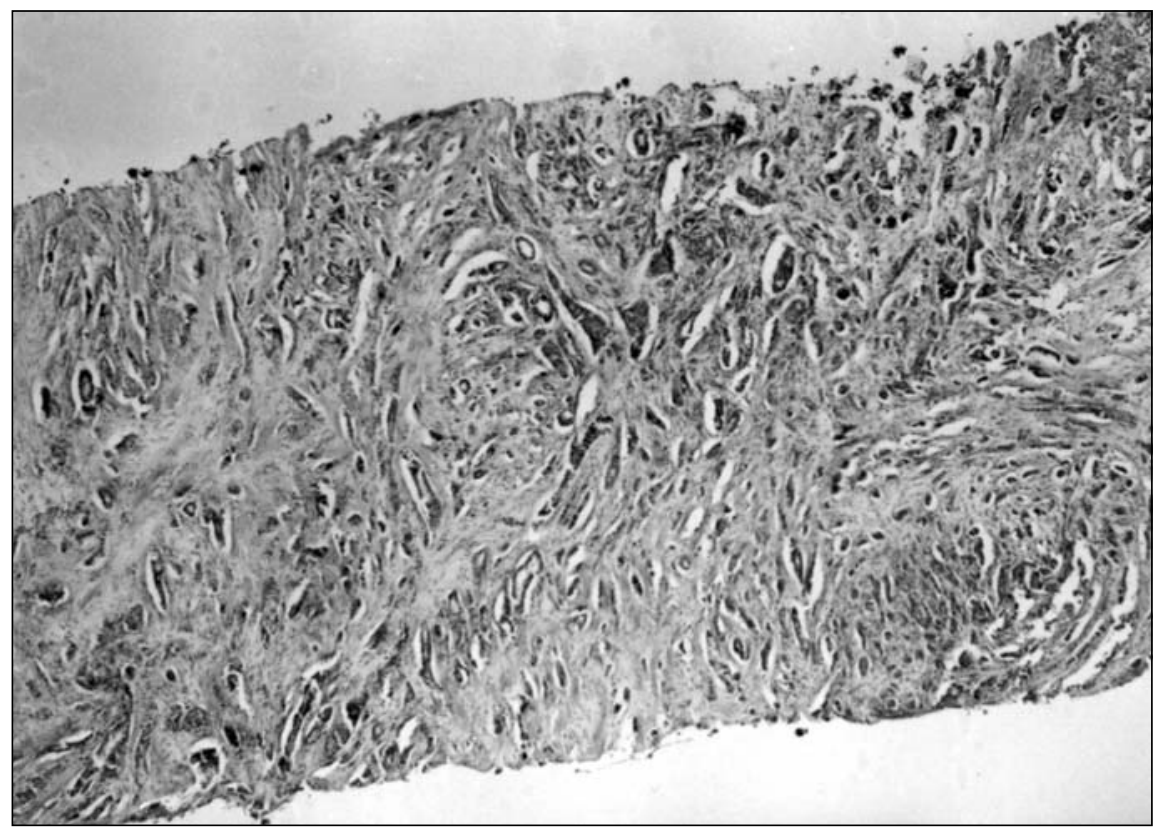

Figura 2. "Core biopsy", agulha 12. Carcinoma ductal invasivo. (Hematoxilina-eosina, original $40 \times$ ).

Não houve diferenças significantes quanto ao número de lesões "borderline" (C), de $32(3,5 \%)$ e dois $(1,8 \%)$ casos para as amostras obtidas por agulhas $14 \mathrm{e} 12$, respectivamente, de um total de 34 lesões diagnosticadas como tais.

\section{DISCUSSÃO}

O número de fragmentos necessários para o diagnóstico histopatológico depende de cada caso e, também, em gran- padas. Parker et al., ao iniciarem o uso de agulhas para obtenção de fragmentos em lesão da mama, começaram utilizando as de calibres 18 e 16, desenvolvidas para a biópsia de próstata ${ }^{(13,14)}$, porém obtiveram amostras muito fragmentadas. Após o aparecimento de agulhas calibre 14 , desenvolvidas especialmente para a mama, conseguiram espécimes adequados para um diagnóstico histológico confiável ${ }^{(15)}$.

Recentemente, vem sendo observada uma tendência à utilização de agulhas mais calibrosas e de equipamentos que permitam a obtenção de maior volume de tecido. Alguns centros estão utilizando unidade de biópsia assistida a vácuo (mamotomia), com a utilização de agulhas calibre 11. Temos experiência ainda limitada no uso do mamótomo. Os autores de relatos sobre a utilização do mamótomo referem, como consequiência do seu uso, uma melhor definição das lesões, dentro do espectro hiperplasia atípica/carcinoma ductal in situ de baixo grau, resultando numa menor proporção de casos cujo diagnóstico de hiperplasia atípica na "core biopsy" foi modificado para carcinoma in situ ou invasivo na peça cirúrgica subseqüente ${ }^{(16,17)}$. Todavia, os melhores resultados obtidos por esse método não eliminam a necessidade de excisão cirúrgica diante de um diagnóstico de hiperplasia atípica ${ }^{(16)}$. A possibilidade de retirada total da lesão pelo mamótomo, que supostamente dificultaria a marcação pré-cirúrgica posterior, tem sido resolvida pela colocação de clipe metálico no sítio da biópsia.

Instrumentos para biópsia percutânea, como o sistema ABBI ("advanced breast biopsy instrumentation"), permitem a retirada de cilindros de tecido com até 2 $\mathrm{cm}$ de diâmetro, removendo as pequenas lesões na sua totalidade ${ }^{(17)}$. Tais equipamentos, porém, não foram bem aceitos, por causa da desvantagem de retirar quantidade excessiva de tecido em lesões benignas, bem como de produzir complicações secundárias que requerem cirurgia posterior.

No momento, todos os instrumentos para biópsia percutânea são considerados de natureza diagnóstica, não sendo admitidos como métodos de tratamento. Nos Estados Unidos, o Food and Drug 
Administration (FDA) chama a atenção para este aspecto.

Portanto, um diagnóstico de carcinoma implica excisão cirúrgica, quaisquer que sejam os tamanhos das amostras e seu método de obtenção. Em bases ainda experimentais, em necropsia (animais e humanos) e in vivo (animais) tem-se usado termoterapia intersticial a "laser", através de fibra óptica guiada por estereotaxia, uma tentativa promissora de futuramente se poder destruir carcinomas mínimos da mama ${ }^{(\mathbf{1 8})}$. No nosso material, como podemos notar na Tabela 1 , mesmo na comparação entre os espécimes obtidos por agulhas 14 houve diferença significativa quanto ao tamanho do maior fragmento ( $p<0,001)$, entre os considerados inadequados e os suficientes para o diagnóstico histopatológico. Esses fragmentos excluídos estavam entre os primeiros obtidos no início da técnica, em 1993.

A utilização de agulhas calibre 12 proporcionou fragmentos com comprimento maior, sendo essa diferença altamente significativa ( $\mathrm{p}<0,001)$ quando comparado com os mesmos parâmetros nas amostras obtidas com agulhas 14 (Tabela 2). A possibilidade de amostra insuficiente/insatisfatória para o diagnóstico é maior com o uso de agulhas menos calibrosas. Assim, na PAAF, em que geralmente são utilizadas agulhas de calibres 22 a 25, observa-se maior porcentual de material insuficiente para o diagnóstico. Nas recomendações da reunião de consenso sobre PAAF, promovida pelo $\mathrm{Na}$ tional Cancer Institute dos EUA e realizada em 1996, em Bethesda, MD ${ }^{(19)}$, recomendou-se manter as taxas de material insuficiente abaixo de $20 \%$, e sugeriu-se a presença do citopatologista no ato da punção, para imediata avaliação, macro e microscópica, da adequação da amostra obtida. $\mathrm{Na}$ "core biopsy" estereotáxica pode-se verificar a quantidade de tecido a olho nu, o que não é possível em material citológico ${ }^{(\mathbf{1 0})}$. $\mathrm{Na}$ avaliação das primeiras 700 "core biopsies" obtidas com agulhas calibre 14 ocorreram apenas 37 casos considerados insuficientes para o diagnóstico $(5,29 \%$ do total), sendo este material significativamente menor no comprimento em relação às amostras consideradas adequadas (Tabela 1).
Há alguma dificuldade na conceituação de "material insuficiente" em biópsia de fragmentos. Não encontramos uma definição na literatura especializada disponível. Embora tenhamos utilizado como critério de exclusão dos 37 casos obtidos por agulhas 14 material contendo apenas pele, estroma conjuntivo, tecido adiposo, sem representação do corpus mammae, temos dúvida se este seria o melhor critério. Esse tipo de material pode representar alterações involutivas do parênquima, mas existe a possibilidade de a amostra não ser representativa da lesão radiológica, não sendo possível o diagnóstico definido em tais casos. $\mathrm{Na}$ verdade, acreditamos que o conceito de "amostra não-representativa" ou de "material insuficiente" só é factível numa abordagem integrada, isto é, com a correlação da radiologia com a anatomia patológica. No material biopsiado por agulhas calibre 12 (109 casos) não ocorreram espécimes considerados insatisfatórios pelo critério adotado.

Como se pode notar no exame do Gráfico 1 e da Tabela 3, predominaram em todas as séries as lesões benignas e, entre elas, as não-neoplásicas.

$\mathrm{Na}$ comparação dos porcentuais das lesões benignas não-neoplásicas nos fragmentos obtidos por agulhas 14 e por agulhas $12(64,7 \%$ vs. $43,11 \%)$ a diferença resultou significativa.

A maior proporção dos casos diagnosticados como benignos não-neoplásicos corresponde a lesões classificadas na categoria 3 do BI-RADS (lesões provavelmente benignas), como demonstrado por Maranhão ${ }^{(\mathbf{1 0})}$ na avaliação das primeiras 433 biópsias obtidas com agulhas 14 .

A redução significativa da frequiência dessas lesões no material obtido com agulhas 12 resulta, provavelmente, de uma maior seleção dos pacientes para a indicação de biópsia percutânea de fragmentos. Verificou-se, ao mesmo tempo, um aumento na proporção de neoplasias benignas quando se compararam as amostras obtidas por agulhas 14 e 12 (14\% vs. $17,43 \%$ ), embora esta diferença não tenha sido significativa.

Maranhão ${ }^{(\mathbf{1 0 )}}$, no estudo do mesmo material acima citado, verificou que $11,1 \%$ das lesões com diagnóstico de neoplasia benigna à "core biopsy" foram classificadas, à mamografia, como categoria 4 (suspeitas de malignidade).

A biópsia estereotáxica é muito eficiente em diagnosticar lesões benignas e excluir malignidade, sendo particularmente útil na avaliação de lesões classificadas como categoria 4, reveladas como benignas após a "core biopsy"(6).

Comparando-se o material obtido pelas agulhas 14 e 12, houve aumento significativo na freqüência de carcinomas nos fragmentos oriundos de agulhas 12 (17,6\% vs. $37,6 \%)$, sendo também significativo o aumento do número de carcinomas in situ $(2,78 \%$ vs. $7,33 \%)$ e invasivos (14,8\% vs. $30,2 \%)$. Esses achados podem refletir maior acurácia no diagnóstico, pela melhor representatividade da amostra, sobretudo no que diz respeito aos carcinomas in situ e, em particular, aos de baixo grau, em que as dificuldades no diagnóstico diferencial com a hiperplasia atípica são bem conhecidas $^{(16,17)}$. Todavia, apesar da maior proporção dos casos de carcinoma in situ nos espécimes obtidos com agulhas 12, não houve diferença significativa no porcentual de lesões "borderline" entre as biópsias obtidas por agulhas 14 e $12(3,56 \%$ vs. $1,83 \%)$. Parece, portanto, que o aumento na proporção de malignos como um todo, e do carcinoma in situ em particular, poderia não se dever à maior representatividade da amostra, nem à melhor definição das lesões dentro do espectro hiperplasia atípica/carcinoma in situ de baixo grau, como parece haver ocorrido com o uso do mamótomo ${ }^{(17)}$. Entretanto, o pequeno número de lesões diagnosticadas como "borderline" (dois casos) na série obtida por agulhas 12 limita esse tipo de conclusão. Os resultados, como aconteceu com as lesões benignas não-neoplásicas, também podem refletir a melhor seleção dos casos pela radiologia, talvez como reflexo da utilização da mamografia digital em campo limitado ("spot" digital) para a realização do método estereotáxico, utilizada em uma grande proporção do material obtido por agulhas 12 .

Não ocorreram falsos-positivos, pois todos os casos de carcinoma foram confirmados na peça cirúrgica. Os dois casos 
diagnosticados como "borderline", nas amostras obtidas com agulhas 12 , foram encaminhados à cirurgia, resultando em um caso de carcinoma intraductal padrão sólido e comedônico, e o outro, de carcinoma ductal invasivo associado a componente intraductal de padrão sólido, cribriforme, micropapilar e lesão esclerosante radial.

Não foi possível avaliar a sensibilidade, nem a especificidade, do método nas amostras de agulhas 12, devido ao curto tempo de acompanhamento clínico-mamográfico das lesões benignas à "core biopsy" (obtidas no período de janeiro/ 1998 a outubro/2000), pois em vários casos ainda não decorreu um período mínimo de seis meses de seguimento.

$\mathrm{Na}$ literatura encontramos apenas o trabalho de Evans et al. $^{(\mathbf{2 0 )}}$ utilizando agulhas calibre 12, comparando a sensibilidade absoluta e a completa no diagnóstico do carcinoma ductal in situ, em microcalcificações mamográficas, não tendo sido constatada melhora no diagnóstico de microcalcificações com a utilização desse calibre. A virtual totalidade dos relatos menciona a utilização de agulhas calibre 14, ou a adoção de agulhas calibre 11, em biópsia assistida a vácuo (mamotomia).

\section{CONCLUSÕES}

A utilização de agulhas calibre 12 para a "core biopsy" estereotáxica de lesões subclínicas da mama:

1) Resultou em maior tamanho de fragmentos, os quais são significativa- mente maiores, e eliminou os casos considerados insuficientes para diagnóstico.

2) Associou-se à redução significativa na frequiência de lesões benignas nãoneoplásicas e ao aumento significativo no porcentual de carcinomas. Este fato não se deve necessariamente ao calibre da agulha, podendo ser resultante do conjunto de critérios que indicaram o procedimento.

\section{REFERÊNCIAS}

1. Abreu-e-Lima MCC. A anatomia patológica nas pequenas biópsias. Vitrô Cancerol 1994;1:20-2.

2. Nath ME, Robinson TM, Tobon H, Chough DM, Sumkin JH. Automated large-core needle biopsy of surgically removed breast lesions - comparison of samples obtained with 14-, 16-, and 18-gauge needles. Radiology 1995;197:739-42.

3. Schnitt SJ. Core needle biopsy: challenges and promises. Handout, US and Canadian Academy of Pathology, 2000 Meeting, 25-31 Mar, New Orleans, 2000:1-6.

4. Abreu-e-Lima MCC. A biópsia estereotática no diagnóstico das lesões impalpáveis da mama. Uma integração da anatomia patológica com a radiologia de alta precisão. [Tese de Doutorado]. Recife: Universidade Federal de Pernambuco, 1997.

5. Abreu-e-Lima MCC, Maranhão N, Costa I, Bulhões TA, Abreu-e-Lima M. Acurácia da "core biopsy" estereotática no diagnóstico de invasão estromal em lesões mamárias impalpáveis. Rev Bras Mastol 1998;8:76-80.

6. Abreu-e-Lima MC, Maranhão N, Costa I, et al. Aumento da especificidade da mamografia no diagnóstico de lesões não-palpáveis: valor da core biopsy estereotática na exclusão de malignidade. J Bras Patol 2000;2:118-23.

7. Abreu-e-Lima MC, Maranhão N, Costa I, Abreue-Lima M, Abreu-e-Lima P, Carvalho ARL. Sensibilidade e especificidade da core biopsy estereotática no diagnóstico histopatológico das lesões mamárias impalpáveis. Rev Ass Med Bras 1999; 45:290-4.

8. Maranhão N,Abreu-e-Lima MCC. A "core biopsy" no diagnóstico das lesões mamárias impalpáveis (Editorial). Radiol Bras 1995;28(2):V.
9. Maranhão N, Costa I, Abreu-e-Lima MC, Nascimento R. A biópsia estereotática no diagnóstico das calcificações mamárias. Radiol Bras 1997;30:12531.

10. Maranhão N. A radiologia intervencionista de alta precisão na detecção de lesões mínimas da mama: estudo de 701 biópsias estereotáticas. [Tese de Doutorado]. Rio de Janeiro: Universidade Federal do Rio de Janeiro, 1999.

11. Liberman L, Dershaw DD, Rosen PP, Abramson AF, Deutch BM, Hann LE. Stereotaxic 14-gauge breast biopsy: how many core biopsy specimens are needed? Radiology 1994;192:793-5.

12. Brenner RJ, Fajardo L, Fisher PR, et al. Percutaneous core biopsy of the breast: effect of operator experience and number of samples on diagnostic accuracy. AJR 1996;166:341-6.

13. Parker SH, Lovin JD, Jobe WE, Burke BJ, Hopper KD, Yakes WF. Nonpalpable breast lesions: stereotactic automated large-core biopsies. Radiology 1991;180:403-7.

14. Parker SH, Lovin JD, Jobe WE, et al. Stereotactic breast biopsy with a biopsy gun. Radiology 1990; 176:741-7.

15. Parker SH, Dennis MA. Core breast biopsy deserves acceptance. Diag Imaging Internat 1994;Jun:39-41.

16. Brem RF, Behrndt VS, Sanow L, Gatewood OMB. Atypical ductal hyperplasia: histologic underestimation of carcinoma in tissue harvested from impalpable breast lesions using 11-gauge stereotactically guided directional vacuum-assisted biopsy. AJR 1999;172:1405-7.

17. Burbank F. Stereotatic breast biopsy of atypical ductal hyperplasia and ductal carcinoma in situ lesions: improved accuracy with directional, vacuum-assisted biopsy. Radiology 1997;202: 843-7.

18. Milne PJ, Parel JM, Manns F, Denham DB, Gonzalez-Cirre X, Robinson DS. Development of stereotactically guided laser interstitial thermotherapy of breast cancer: in situ measurement and analysis of the temperature field in ex vivo and in vivo adipose tissue. Lasers Surg Med 2000;26:6775.

19. National Cancer Institute. The uniform approach to breast fine needle aspiration biopsy - a synopsis. Acta Cytol 1996;40:1120-6.

20. Evans AJ, Whitlock JP, Burrel HC, et al. A comparison of 14- and 12-gauge needles for core biopsy of suspicious mammographic calcification. Br J Radiol 1999;72:1152-4. 